\title{
WEAKLY COMPACT SETS
}

\author{
BY \\ ROBERT C. JAMES(1)
}

It has been conjectured that a closed convex subset $C$ of a Banach space $B$ is weakly compact if and only if each continuous linear functional on $B$ attains a maximum on $C$ [5]. This reduces easily to the case in which $C$ is bounded, and will be answered in the affirmative [Theorem 4] after some preliminary results are established. Following suggestions by Namioka and Peck, the result is then generalized, first to weakly closed subsets of Banach spaces and then to weakly closed subsets of complete locally convex linear spaces.

The original motivation for this conjecture was the knowledge that it is true when $C$ is the unit sphere of a separable Banach space [2], which was later extended to arbitrary Banach spaces [3, Theorem 5]. Additional support was given the conjecture when V. L. Klee [5] proved a seemingly related theorem-namely, that if $C$ is a bounded closed non-weakly-compact convex subset of a Banach space, then there is a decreasing sequence $\left\{K_{i}\right\}$ of nonempty closed convex subsets of $C$ such that, for each $x \in C$ and each $m \in[0,1)$, the set $x+m(C-x)$ meets only finitely many of the sets $\left\{K_{i}\right\}$. If $f$ is a continuous linear functional whose sup on $C$ is $M$ and if there is no $x$ in $C$ for which $f(x)=M$, then a suitable choice for Klee's sequence $\left\{K_{i}\right\}$ is to let $K_{n}=C \cap\{x: f(x) \geqq M-1 / n\}$ for each $n$.

It is interesting to note that the conjecture can be verified easily for a bounded closed convex set that is symmetric about an interior point $x$, since if $x$ is translated to 0 , then the convex set as a unit sphere induces a norm for which the new Banach space is isomorphic to the original space. More generally, if 0 is an interior point of a bounded convex set $C$ and $K$ is the closed convex span of $C \cup(-C)$, then $K$ is symmetric about 0 and the sup on $K$ of a continuous linear functional is the larger of its sups on $C$ and $-C$. Therefore for convex bodies the conjecture can be established by using the known theorem for unit spheres [3, Theorem 5, p. 215].

The following theorem is a generalization of a characterization of weak compactness of the unit sphere that was useful in [2]. In the proof of this theorem and thereafter, we shall use the convention that a sequence of nonoverlapping

Presented to the Society, April 2, 1963 ; received by the editors April 22, 1963.

(1) This work was supported in part by National Science Foundation grant number NSFGP-192. 
members of conv $\left\{y_{n}\right\}$ is a sequence $\left\{x_{n}\right\}$ for which there is an increasing sequence of integers $\left\{p_{i}\right\}$ such that $x_{n}$ belongs to conv $\left\{y_{p_{n}}, \cdots, y_{p_{n+1}-1}\right\}$ for all $n$.

THEOREM 1. A bounded closed convex subset $C$ of a Banach space is not weakly compact if and only if there exist positive numbers $\mu$ and $\sigma$ such that if $\delta$ and $\Delta$ are any numbers with $\delta<\mu<\Delta$, then there exists a sequence $\left\{z_{i}\right\}$ of members of $C$ for which the following are true for all positive integers $n$ :

(i) $\delta<\|\xi\|<\Delta$ for all $\xi \in \operatorname{conv}\left\{z_{i}\right\}$;

(ii) $d\left(\operatorname{conv}\left\{z_{i}, \cdots, z_{n}\right\}\right.$, conv $\left.\left\{z_{n+1}, \cdots\right\}\right) \geqq \sigma$.

Proof. It is known that a closed convex subset $C$ of a Banach space is weakly compact if and only if each decreasing sequence $\left\{K_{i}\right\}$ of closed convex sets contained in $C$ has a nonempty intersection $[1 ; 6]$. If $\left\{z_{i}\right\}, \delta, \Delta$, and $\sigma$ exist for which (i) and (ii) are satisfied, we can let $K_{n}$ be cl [ $\left.\operatorname{conv}\left\{z_{n}, z_{n+1}, \cdots\right\}\right]$ for each $n$. If $w \in K_{n}$ for all $n$, then there are nonoverlapping members $\eta$ and $\zeta$ of $\operatorname{conv}\left\{z_{i}\right\}$ such that

$$
\|w-\eta\|<\frac{1}{2} \sigma \text { and }\|w-\zeta\|<\frac{1}{2} \sigma .
$$

Then $\|\eta-\zeta\|<\sigma$. But it follows from (ii) that $\|\eta-\zeta\| \geqq \sigma$. Therefore the sequence $\left\{K_{i}\right\}$ has an empty intersection and $C$ is not weakly compact.

Now suppose that $C$ is bounded, closed, convex, and not weakly compact. Then there is a decreasing sequence $\left\{T_{i}\right\}$ of closed convex subsets of $C$ that have an empty intersection. Choose a sequence $\left\{t_{n}\right\}$ for which $t_{n} \in T_{n}$ for each $n$. We shall show first that if it is not possible to satisfy (ii) with $\sigma=\frac{1}{3}$ and $\left\{z_{i}\right\}$ some sequence of nonoverlapping members of conv $\left\{t_{i}\right\}$, then there is a member $t_{1}^{1}$ of conv $\left\{t_{i}\right\}$ for which there is a sequence $\left\{t_{i}^{1}\right\}$ of nonoverlapping members of conv $\left\{t_{i}\right\}$ such that

$$
\left\|t_{1}^{1}-t_{n}^{1}\right\|<\frac{1}{2} \text { for all } n>1 .
$$

To show this, we can suppose that no such $t_{1}^{1}$ exists. Then we let $z_{1}=t_{1}$. There is then an $n_{1}$ such that $\left\|z_{1}-w\right\| \geqq \frac{1}{2}$ for all $w$ in conv $\left\{t_{n_{1}}, t_{n_{1}+1}, \cdots\right\}$. Let $z_{2}$ $=t_{n_{1}}$. For any finite set $M$ of members of $\operatorname{conv}\left\{z_{1}, z_{2}\right\}$, we can choose $n_{2}$ large enough that $\|z-w\| \geqq \frac{1}{2}$ for all $z$ in $M$ and all $w$ in conv $\left\{t_{n_{2}+1}, t_{n_{2}+2}, \cdots\right\}$. Therefore we can choose $n_{2}$ so that $\|z-w\| \geqq \frac{1}{2}$ for all $z$ in $\operatorname{conv}\left\{z_{1}, z_{2}\right\}$ and all $w$ in conv $\left\{t_{n_{2}+1}, t_{n_{2}+2}, \cdots\right\}$. Continuing in this way, we could satisfy (ii) with $\sigma=\frac{1}{2}$. If it is not true that (ii) can be satisfied for some $\sigma>0$ and some sequence of nonoverlapping members of conv $\left\{t_{n}\right\}$, then an argument similar to that just completed can be used inductively to obtain sequences $\left\{t_{n}^{k}\right\}, k=1,2, \cdots$, such that $\left\{t_{n}^{k}\right\}$ is a sequence of nonoverlapping members of conv $\left\{t_{2}^{k-1}, t_{3}^{k-1}, \cdots\right\}$ and

$$
\left\|t_{1}^{k}-t_{n}^{k}\right\|<\frac{1}{k+1} \text { for all } n
$$


Then $\left\|t_{1}^{k}-t_{1}^{p}\right\|<1 /(k+1)$ for all $p>k$, so $\left\{t_{1}^{k}\right\}$ is a Cauchy sequence. Its limit is in the intersection of the sets $\left\{T_{n}\right\}$, contrary to assumption. Therefore we can conclude that there is a positive number $\sigma$, and a sequence $\left\{z_{n}^{*}\right\}$ of nonoverlapping members of conv $\left\{t_{n}\right\}$, for which (ii) is satisfied.

Now let $\mu=\lim _{n \rightarrow \infty} \inf \left\{\|x\|: x \in \operatorname{conv}\left\{z_{n}^{*}, z_{n+1}^{*}, \cdots\right\}\right\}$. Since 0 is not in the intersection of the sets $\left\{T_{i}\right\}$, we have $\mu>0$. If $\delta<\mu<\Delta$, we can choose $N$ so that

$$
\delta<\inf \left\{\|x\|: x \in \operatorname{conv}\left\{z_{N}^{*}, z_{N+1}^{*}, \cdots\right\}\right\} .
$$

Now choose $\left\{z_{i}\right\}$ so that for each $n$ we have $\left\|z_{n}\right\|<\Delta$ and $\left\{z_{i}\right\}$ is a sequence of nonoverlapping members of $\operatorname{conv}\left\{z_{N}^{*}, z_{N+1}^{*}, \cdots\right\}$. If $\xi \in \operatorname{conv}\left\{z_{i}\right\}$, then $\delta<\|\xi\|$ $<\Delta$, so that (i) is now satisfied. Also, (ii) is satisfied by $\left\{z_{i}\right\}$ since (ii) was satisfied by $\left\{z_{i}^{*}\right\}$.

It is possible to strengthen Theorem 1 by replacing conv $\left\{z_{1}, \cdots, z_{n}\right\}$ in (ii) by the linear span of $\left\{z_{1}, \cdots, z_{n}\right\}$, denoted by lin $\left\{z_{1}, \cdots, z_{n}\right\}$. This is a consequence of the following theorem.

THEOREM 2. Suppose that for a sequence $\left\{z_{i}\right\}$ and positive numbers $\delta$ and $\sigma$, we have

(i) $\delta<\|\xi\|$ for all $\xi \in \operatorname{conv}\left\{z_{i}\right\}$,

(ii) $d$ (conv $\left\{z_{1}, \cdots, z_{n}\right\}$, conv $\left.\left\{z_{n+1}, \cdots\right\}\right) \geqq \sigma$ for all $n$.

If $\theta<\frac{1}{2} \sigma$, then there is a subsequence $\left\{z_{i}^{*}\right\}$ of $\left\{z_{i}\right\}$ such that

(ii) $^{\prime} d\left(\right.$ lin $\left\{z_{1}^{*}, \cdots, z_{n}^{*}\right\}$, conv $\left.\left\{z_{n+1}^{*}, \cdots\right\}\right) \geqq \theta$ for all $n$.

Proof. Suppose that $\theta<\frac{1}{2} \sigma$. We shall use induction to show the existence of the subsequence $\left\{z_{i}^{*}\right\}$. Suppose that a positive integer $p_{k}$ and a finite set $\left\{z_{1}^{*}, z_{2}^{*}, \cdots, z_{k}^{*}\right\}$ have been chosen so that (ii)' is valid for $n \leqq k$ if we use for $\left\{z_{i}^{*}\right\}$ the sequence consisting of $\left\{z_{1}^{*}, \cdots, z_{k}^{*}\right\}$ and all $z_{i}$ with $i>p_{k}$. If the analogous statement is not true for $n \leqq k+1$ when $z_{k+1}^{*}=z_{p_{k}}$ and $p_{k+1}$ is sufficiently large, then for any $p$ there are members $u$ and $z$ of lin $\left\{z_{1}^{*}, \cdots, z_{k+1}^{*}\right\}$ and $\operatorname{conv}\left\{z_{p}, z_{p+1}, \cdots\right\}$ such that

$$
\|u-z\|<\theta .
$$

Since $p$ can be arbitrarily large, there are an infinite number of nonoverlapping choices for $z$. It follows from this and $k$ being fixed that for any positive number $\varepsilon$ there are members $u_{1}$ and $u_{2}$ of lin $\left\{z_{1}^{*}, \cdots, z_{k+1}^{*}\right\}$, and nonoverlapping members $\eta$ and $\zeta$ of conv $\left\{z_{k+2}^{*}, \cdots\right\}$, for which

$$
\left\|u_{1}-\eta\right\|<\theta, \quad\left\|u_{2}-\zeta\right\|<\theta,
$$

and

$$
\left\|u_{1}-u_{2}\right\|<\varepsilon .
$$

Then $\|\eta-\zeta\|<2 \theta+\varepsilon$. It follows from this and (ii) that 


$$
2 \theta+\varepsilon>\sigma \text { and } \theta>\frac{1}{2} \sigma-\frac{1}{2} \varepsilon .
$$

Since $\varepsilon$ was arbitrary, we have $\theta \geqq \frac{1}{2} \sigma$. This contradicts the assumption that $\theta<\frac{1}{2} \sigma$, so we conclude that the induction can be completed.

The next theorem is the particular consequence of Theorems 1 and 2 that will be used in proving Theorem 4. It is a generalization of a similar theorem about nonreflexive Banach spaces-Theorem 1 of [3].

THEOREM 3. A bounded closed convex subset $C$ of a Banach space is not weakly compact if and only if there is a positive number $r$ for which there exists a sequence $\left\{z_{i}\right\}$ of members of $C$, and a sequence $\left\{f_{i}\right\}$ of linear functionals with unit norms, such that $f_{n}\left(z_{i}\right)>r$ if $n \leqq i$ and $f_{n}\left(z_{\imath}\right)=0$ if $n>i$.

Proof. Suppose first that such sequences $\left\{z_{i}\right\}$ and $\left\{f_{i}\right\}$ exist. Let $K_{n}$ be $\operatorname{cl}\left[\operatorname{conv}\left\{z_{n}, z_{n+1}, \cdots\right\}\right]$. Then the decreasing sequence $\left\{K_{i}\right\}$ of closed convex subsets of $C$ has an empty intersection, since if $w$ belongs to the intersection, then $f_{n}(w) \geqq r$ for all $n$ and $\lim _{n \rightarrow \infty} f_{n}(w)=0$. Therefore $C$ is not weakly compact.

Suppose now that $C$ is bounded, closed, convex, and not weakly compact. Then it follows from Theorems 1 and 2 that there is a sequence $\left\{z_{i}\right\}$ of members of $C$, and positive numbers $\delta, \Delta$, and $r$, such that

(i)* $\delta<\|\xi\|<\Delta$ for all $\xi \in \operatorname{conv}\left\{z_{i}\right\}$;

(ii)* $d$ (lin $\left\{z_{i}, \cdots, z_{n}\right\}$, conv $\left.\left\{z_{n+1}, \cdots\right\}\right) \geqq r$ for all $n$.

As a first step toward defining the desired continuous linear functional $f_{n}$ for a particular $n$, let us define a new norm on $\operatorname{lin}\left\{z_{i}\right\}$ as follows: Let $S_{n-1}$ denote lin $\left\{z_{1}, \cdots, z_{n-1}\right\}$ and, when $z=\sum a_{i} z_{i}$, let

$$
\|z\|=\max \left[d\left(z, S_{n-1}\right),\left\|\sum_{1}^{n-1} a_{i} z_{i}\right\|\right] .
$$

If $z \in S_{n-1}$, then $\||| z \mid\|=\|z\|$. If $\||z|\|=0$, then we must have $d\left(z, S_{n-1}\right)=0$ and $z \in S_{n-1}$, so that $\|z \mid\|=\|z\|$. Therefore $\|z\| \neq 0$ if $z \neq 0$. The triangle inequality is satisfied by \|\|$\|$, since it is satisfied by $p$ if either $d\left(z, S_{n-1}\right)$ is used for $p(z)$ or $\left\|\sum_{1}^{n-1} a_{i} z_{i}\right\|$ is used for $p(z)$ when $z=\sum a_{i} z_{i}$. Now let

$$
\frac{1}{\theta}=\inf \left\{|||z|||: z \in \operatorname{conv}\left\{z_{n}, z_{n+1}, \cdots\right\}\right\} .
$$

It follows from the definition of ||| ||| and (ii)* that

$$
\frac{1}{\theta}=d\left(S_{n-1}, \operatorname{conv}\left\{z_{n}, z_{n+1}, \cdots\right\}\right) \geqq r .
$$

The definition of $\theta$ implies that $\operatorname{conv}\left\{\theta z_{n}, \theta z_{n+1}, \cdots\right\}$ contains no points $x$ of lin $\left\{z_{n}, z_{n+1}, \cdots\right\}$ for which $\||x|\|<1$. Therefore [7], there is a linear functional $f_{n}^{*}$ whose domain is cl $\left[\operatorname{lin}\left\{z_{n}, z_{n+1}, \cdots\right\}\right]$ and for which 


$$
f_{n}^{*}(z) \geqq 1 \text { for all } z \in \operatorname{conv}\left\{\theta z_{n}, \theta z_{n+1}, \cdots\right\} \text {, }
$$

and

$$
f_{n}^{*}(u) \leqq 1 \text { if }\|\| u \| \leqq 1
$$

Let $f_{n}^{* *}$ be defined by $f_{n}^{* *}(x)=f_{n}^{*}(x)$ when $x$ is in the domain of $f_{n}^{*}$, and $f_{n}^{* *}\left(z_{i}\right)=0$ if $i<n$. Then

$$
\left|f_{n}^{* *}\left(\sum a_{i} z_{i}\right)\right|=\left|f_{n}^{*}\left(\sum_{n} a_{i} z_{i}\right)\right| \leqq\left\||| \sum_{n} a_{i} z_{i} \mid\right\| .
$$

Since $\mid\left\|\sum_{n} a_{i} z_{i}\right\|=d\left(\sum_{n} a_{i} z_{i}, S_{n-1}\right) \leqq\left\|\sum a_{i} z_{i}\right\|$, we have

$$
\left|f_{n}^{* *}\left(\sum a_{i} z_{i}\right)\right| \leqq\left\|\Sigma a_{i} z_{i}\right\| \text {. }
$$

Therefore $\left\|f_{n}^{* *}\right\| \leqq 1$. Also, if $n \leqq i$, it follows from (3) that $f_{n}^{*}\left(\theta z_{i}\right) \geqq 1$, and from this and (2) that

$$
f_{n}^{* *}\left(z_{i}\right)=f_{n}^{*}\left(z_{i}\right) \geqq \frac{1}{\theta} \geqq r .
$$

Now we can define $f_{n}$ as a norm-preserving extension of $f_{n}^{* *} /\left\|f_{n}^{* *}\right\|$ to the entire space.

The second of the following lemmas is similar to the lemma used in [3] to prove that if a Banach space is nonreflexive then there is a continuous linear functional that does not attain its sup on the unit sphere. However, that lemma and the associated theorem were constructed by analogy with Theorem 2 of [3], which states that there is a continuous linear functional $g$ on $l^{(1)}$ which has the property that, if $l^{(1)}$ is a subspace of a Banach space $B$, then there is a norm-preserving extension of $g$ that does not attain its sup on the unit sphere of $B$. Lemma 2 and Theorem 4 use an analogy to continuous linear functionals of type $\sum \beta_{i} x_{i}$ defined on $\left(c_{0}\right)$, where $\beta_{i}>0$ for each $i$ and $\sum \beta_{i}<+\infty$. Such a functional does not attain its sup on the unit sphere of $\left(c_{0}\right)$.

If $B$ is separable, then the following lemmas and theorem can be simplified as follows. First choose a subsequence $\left\{f_{p_{i}}\right\}$ of $\left\{f_{i}\right\}$ for which $\lim f_{p_{i}}(x)$ exists for each $x$ of $B$, and let $f_{n}^{*}$ be defined as $h_{n} /\left\|h_{n}\right\|$, where

$$
h_{n}(x)=f_{p_{n}}(x)-\lim \left[f_{p_{i}}(x)\right]
$$

for each $x$ of $B$. Then for the sequences $\left\{f_{i}\right\}$ and $\left\{z_{i}\right\}$ of Theorem 3 , there is a subsequence $\left\{z_{i}^{*}\right\}$ of $\left\{z_{i}\right\}$ such that $f_{n}^{*}\left(z_{i}^{*}\right)>\frac{1}{2} r$ if $n \leqq i$ and $f_{n}^{*}\left(z_{i}^{*}\right)=0$ if $n>i$. If $\left\{f_{i}^{*}\right\},\left\{z_{i}^{*}\right\}$, and $\frac{1}{2} r$ are used instead of $\left\{f_{i}\right\},\left\{z_{i}\right\}$ and $r$, then Lemma 1 can be discarded and every lim inf and lim sup used in the proofs of Lenmma 2 and Theorem 4 is equal to zero.

LEMMA 1. Let $C$ be a bounded convex subset of a Banach space $B$ and let $g$ and $\bar{G}_{1}, \bar{G}_{2}, \cdots$ be continuous linear functionals on $B$. If $\beta, \gamma$, and $\varepsilon$ are nonnegative numbers, then there exists a sequence $\left\{G_{i}\right\}$ of nonoverlapping members of conv $\left\{\bar{G}_{i}\right\}$ such that 


$$
\begin{aligned}
& \sup _{x \in C}[g(x)\left.+\beta h_{1}(x)-\gamma \liminf h_{i}(x)\right] \\
&<\varepsilon+\sup _{x \in C}\left[g(x)+\beta h_{1}(x)-\gamma \lim \sup h_{i}(x)\right]
\end{aligned}
$$

for all sequences $\left\{h_{i}\right\}$ of nonoverlapping members of conv $\left\{G_{i}\right\}$.

Proof. Let us first arrange all members of conv $\left\{\bar{G}_{i}\right\}$ that have rational coefficients in a sequence $\left\{\phi_{i}\right\}$. Choose $\left\{F_{i}\right\}$ and an element $x_{1}$ of $C$ so that $\left\{F_{i}\right\}$ is a sequence of nonoverlapping members with rational coefficients of conv $\left\{\bar{G}_{i}\right\}$ which has the property that

$$
g\left(x_{1}\right)+\beta \phi_{1}\left(x_{1}\right)-\gamma \lim \inf F_{i}\left(x_{1}\right)
$$

is within $\frac{1}{2} \varepsilon$ of being as large as possible for all such sequences and elements $x_{1}$. Then let $\left\{F_{i}^{1}\right\}$ be a subsequence of $\left\{F_{i}\right\}$ for which $\lim F_{i}^{1}\left(x_{1}\right)$ exists and equals $\lim \inf F_{i}\left(x_{1}\right)$. Now use this process inductively to choose for each $k$ a sequence $\left\{F_{i}^{k}\right\}$ and an element $x_{k}$ of $C$ with the following properties:

(a) $\left\{F_{i}^{k}\right\}$ is a sequence of nonoverlapping members with rational coefficients of $\operatorname{conv}\left\{F_{2}^{k-1}, F_{3}^{k-1}, \cdots\right\}$;

(b) $\left[g\left(x_{k}\right)+\beta \phi_{k}\left(x_{k}\right)-\gamma \lim \inf _{i \rightarrow \infty} F_{i}^{k}\left(x_{k}\right)\right]$ is within $\frac{1}{2} \varepsilon$ of being as large as possible for all choices of $\left\{F_{i}^{k}\right\}$ as a sequence of nonoverlapping members of conv $\left\{F_{2}^{k-1}, F_{3}^{k-1}, \cdots\right\}$ and all choices of $x_{k}$ from $C$;

(c) $\lim _{i \rightarrow \infty} F_{i}^{k}\left(x_{k}\right)$ exists.

Now let $G_{k}=F_{1}^{k}$ for each $k$. For any $\phi_{k}$ in conv $\left\{G_{i}\right\}$, the expression

$$
g\left(x_{k}\right)+\beta \phi_{k}\left(x_{k}\right)-\gamma \liminf G_{i \rightarrow \infty}\left(x_{k}\right)=g\left(x_{k}\right)+\beta \phi_{k}\left(x_{k}\right)-\gamma \lim _{i \rightarrow \infty} F_{i}^{k}\left(x_{k}\right),
$$

and this expression cannot be increased by more than $\frac{1}{2} \varepsilon$ by replacing $\left\{G_{i}\right\}$ by some sequence $\left\{h_{i}\right\}$ of nonoverlapping members of conv $\left\{G_{i}\right\}$ and $x_{k}$ by some member of $C$. Also, $\lim \inf _{i \rightarrow \infty} h_{i}\left(x_{k}\right)=\lim \sup _{i \rightarrow \infty} h_{i}\left(x_{k}\right)$ for any such sequence. Now for a choice of $\left\{h_{i}\right\}$, we can choose $\phi_{k}$ so that $\left|h_{1}(x)-\phi_{k}(x)\right|<\frac{1}{4} \varepsilon$ if $x \in C$ and use $\phi_{k}$ and the corresponding $x_{k}$ to show that it is impossible to have

$$
\begin{aligned}
& \sup _{x \in C}\left[g(x)+\beta h_{1}(x)-\gamma \lim \inf h_{i}(x)\right] \\
& \geqq \varepsilon+\sup _{x \in C}\left[g(x)+\beta h_{1}(x)-\gamma \lim \sup h_{i}(x)\right],
\end{aligned}
$$

since we could then replace $h_{1}$ by $\phi_{k}$ and obtain

$$
\left[g\left(x_{k}\right)+\beta \phi_{k}\left(x_{k}\right)-\gamma \operatorname{lim\operatorname {sup}} h_{i}\left(x_{k}\right)\right]>\sup _{x \in C}\left[g(x)+\beta \phi_{k}(x)-\gamma \lim \sup h_{i}(x)\right] .
$$

LEMMA 2. Suppose that $C$ is a bounded convex subset of a Banach space $B$, $r$ is a positive number, $\left\{z_{i}\right\}$ is a sequence of members of $C$, and $\left\{f_{i}\right\}$ is a sequence of linear functionals with unit norms such that $f_{n}\left(z_{i}\right)>r$ if $n \leqq i$ and $f_{n}\left(z_{i}\right)=0$ if $n>i$. If $\left\{\beta_{i}\right\}$ is a sequence of positive numbers, then there is a sequence $\left\{g_{i}\right\}$ of nonoverlapping members of conv $\left\{f_{i}\right\}$ which has the following property $\mathrm{K}$ : 
If $g_{k}(\xi)-\lim \inf g_{i}(\xi) \leqq\left(\frac{1}{4}+2^{-k}\right) r$ for some $k$ and some $\xi \in C$, then there is $a$ $y$ in $C$ such that

$$
\begin{aligned}
& {\left[\sum_{1}^{k} \beta_{i} g_{i}(y)-\left(\sum_{1}^{k} \beta_{i}\right) \lim \sup g_{i}(y)\right]} \\
& >2^{-(k+2)} r \beta_{k}+\left[\sum_{1}^{k} \beta_{i} g_{i}(\xi)-\left(\sum_{1}^{k} \beta_{i}\right) \liminf g_{i}(\xi)\right]
\end{aligned}
$$

Proof. We shall show first that $\left\{g_{i}\right\}$ has property $\mathrm{K}$ when $k=1$, if $\left\{g_{i}\right\}$ is any sequence of nonoverlapping members of $\operatorname{conv}\left\{f_{i}\right\}$. To do this, we suppose that $\left\{g_{i}\right\}$ has been chosen and that a member $\xi$ of $C$ has the property that

$$
g_{1}(\xi)-\lim \inf g_{i}(\xi) \leqq \frac{3}{4} r
$$

We have

$$
\beta_{1} g_{1}\left(z_{n}\right)-\beta_{1} \lim \sup g_{i}\left(z_{n}\right)=\beta_{1} g_{1}\left(z_{n}\right)>r \beta_{1}
$$

if $n$ is large enough. Therefore there is an $n$ large enough that we can satisfy (4) with $k=1$ and $y=z_{n}$ if it is true that

$$
r \beta_{1}>\frac{1}{8} r \beta_{1}+\beta_{1}\left[g_{1}(\xi)-\lim \inf g_{i}(\xi)\right]
$$

This inequality is true, since $g_{1}(\xi)-\lim \inf g_{i}(\xi) \leqq \frac{3}{4} r$.

Now suppose that $\left\{g_{1}, g_{2}, \cdots, g_{n-1}, F_{n}, F_{n+1}, \cdots\right\}$ has been chosen as a sequence of nonoverlapping members of conv $\left\{f_{i}\right\}$ and that, for each sequence $\left\{g_{n}, g_{n+1}, \cdots\right\}$ of nonoverlapping members of conv $\left\{F_{n}, F_{n+1}, \cdots\right\}$, property $\mathrm{K}$ is satisfied when $k \leqq n$. Let

$$
\theta_{n}=\inf _{\left\{G_{i}\right\}} \sup _{x \in C}\left[\sum_{1}^{n-1} \beta_{i} g_{i}(x)+\beta_{n} G_{n}(x)-\left(\sum_{1}^{n} \beta_{i}\right) \lim \inf G_{i}(x)\right],
$$

where $\left\{G_{i}\right\}$ denotes a sequence $\left\{G_{n}, G_{n+1}, \cdots\right\}$ of nonoverlapping members of $\operatorname{conv}\left\{F_{n}, F_{n+1}, \cdots\right\}$. Choose a particular $\left\{G_{i}^{*}\right\}$ so that

$$
\sup _{x \in C}\left[\sum_{1}^{n-1} \beta_{i} g_{i}(x)+\beta_{n} \bar{G}_{n}^{*}(x)-\left(\sum_{1}^{n} \beta_{i}\right) \lim \inf G_{i}^{*}(x)\right]<\theta_{n}+2^{-(n+3)} r \beta_{n+1} .
$$

If $G_{n}^{*}=G_{n}^{*}$ and $\left\{G_{n+1}^{*}, \cdots\right\}$ is a sequence of nonoverlapping members of $\operatorname{conv}\left\{\bar{G}_{n+1}^{*}, \cdots\right\}$, then for all sequences $\left\{h_{n+1}, \cdots\right\}$ of nonoverlapping members of $\operatorname{conv}\left\{G_{n+1}^{*}, \cdots\right\}$ we have $\liminf h_{i}(x) \geqq \liminf G_{i}^{*}(x)$ for all $x$ in $B$. Therefore it follows from Lemma 1 that we can let $G_{n}^{*}=G_{n}^{*}$ and choose $\left\{G_{n+1}^{*}, \cdots\right\}$ so that

(6) $\sup _{x \in \mathcal{C}}\left[\sum_{1}^{n-1} \beta_{i} g_{i}(x)+\beta_{n} G_{n}^{*}(x)-\left(\sum_{1}^{n} \beta_{i}\right) \liminf h_{i}(x)\right]<\theta_{n}+2^{-(n+3)} r \beta_{n+1}$, 


$$
\begin{aligned}
\sup _{x \in C} & {\left[\sum_{1}^{n-1} \beta_{i} g_{i}(x)+\beta_{n} G_{n}^{*}(x)+\beta_{n+1} h_{n+1}(x)-\left(\sum_{1}^{n+1} \beta_{i}\right) \liminf h_{i}(x)\right] } \\
& <2^{-(n+3)} r \beta_{n+1} \\
+ & \sup _{x \in C}\left[\sum_{1}^{n-1} \beta_{i} g_{i}(x)+\beta_{n} G_{n}^{*}(x)+\beta_{n+1} h_{n+1}(x)-\left(\sum_{1}^{n+1} \beta_{i}\right) \limsup h_{i}(x)\right],
\end{aligned}
$$

for all sequences $\left\{h_{n+1}, \cdots\right\}$ of nonoverlapping members of $\operatorname{conv}\left\{G_{n+1}^{*}, \cdots\right\}$.

Now suppose there exists a $G^{* *}$ in $\operatorname{conv}\left\{G_{n+1}^{*}, \cdots\right\}$ and a sequence $\left\{h_{n+1}, \cdots\right\}$ of nonoverlapping members of $\operatorname{conv}\left\{G_{n+1}^{*}, \cdots\right\}$ for which

$$
\sup _{x \in C}\left[\sum_{1}^{n-1} \beta_{i} g_{i}(x)+\beta_{n} G_{n}^{*}(x)+\beta_{n+1} G^{* *}(x)-\left(\sum_{1}^{n+1} \beta_{i}\right) \liminf h_{i}(x)\right]
$$

$$
<\theta_{n}+\left(\frac{1}{4}+2^{-n}\right) r \beta_{n+1} \text {. }
$$

Let $H=\left(\beta_{n} G_{n}^{*}+\beta_{n+1} G^{* *}\right) /\left(\beta_{n}+\beta_{n+1}\right)$. Then

$$
\begin{aligned}
\sup _{x \in C}\left[\sum_{1}^{n-1} \beta_{i} g_{i}(x)+\left(\beta_{n}+\beta_{n+1}\right) H(x)-\left(\sum_{1}^{n+1} \beta_{i}\right) \liminf h_{i}(x)\right] \\
<\theta_{n}+\left(\frac{1}{4}+2^{-n}\right) r \beta_{n+1} .
\end{aligned}
$$

We know that property $\mathrm{K}$ is valid for $k=n$ with $h_{i}$ substituted for $g_{i}$ when $i>n$ and $H$ substituted for $g_{n}$. Therefore if $\xi \in C$ is such that the expression $S$,

$$
S=\sum_{1}^{n-1} \beta_{i} g_{i}(x)+\beta_{n} H(x)-\left(\sum_{1}^{n} \beta_{i}\right) \liminf h_{i}(x),
$$

is within $2^{-(n+2)} r \beta_{n}$ of its sup when $x=\xi$, then

$$
H(\xi)-\liminf h_{i}(\xi)>\left(\frac{1}{4}+2^{-n}\right) r
$$

and $S$ is increased by more than $\left(\frac{1}{4}+2^{-n}\right) r \beta_{n+1}$ if the quantity

$$
\beta_{n+1}\left[H(\xi)-\liminf h_{i}(\xi)\right]
$$

is added, so it follows from (9) that

$$
\begin{aligned}
\sup _{x \in C}\left[\sum_{1}^{n-1} \beta_{i} g_{i}(x)+\beta_{n} H(x)-\left(\sum_{1}^{n} \beta_{i}\right) \liminf h_{i}(x)\right] \\
<\theta_{n}+\left(\frac{1}{4}+2^{-n}\right) r \beta_{n+1}-\left(\frac{1}{4}+2^{-n}\right) r \beta_{n+1}=\theta_{n} .
\end{aligned}
$$

This contradicts (5), so we can conclude that (8) is false for all $G^{* *}$ and all sequences $\left\{h_{n+1}, \cdots\right\}$ of nonoverlapping members of $\operatorname{conv}\left\{G_{n+1}^{*}, \cdots\right\}$. It follows from this and (6) that 


$$
\begin{aligned}
\sup _{x \in C} & {\left[\sum_{1}^{n-1} \beta_{i} g_{i}(x)+\beta_{n} G_{n}^{*}(x)+\beta_{n+1} G^{* *}(x)-\left(\sum_{1}^{n+1} \beta_{i}\right) \liminf h_{i}(x)\right] } \\
> & {\left[\frac{1}{4}+2^{-n}-2^{-(n+3)}\right] r \beta_{n+1} } \\
& +\sup _{x \in C}\left[\sum_{1}^{n-1} \beta_{i} g_{i}(x)+\beta_{n} G_{n}^{*}(x)-\left(\sum_{1}^{n} \beta_{i}\right) \liminf h_{i}(x)\right],
\end{aligned}
$$

whatever the choice of $G^{* *}$ and $\left\{h_{n+1}, \cdots\right\}$ as nonoverlapping members of $\operatorname{conv}\left\{G_{n+1}^{*}, \cdots\right\}$. Now let $G_{n}^{*}=g_{n}$. It then follows from (7) and (10) that

$$
\begin{aligned}
& \sup _{x \in C}\left[\sum_{1}^{n} \beta_{i} g_{i}(x)+\beta_{n+1} h_{n+1}(x)-\left(\sum_{1}^{n+1} \beta_{i}\right) \limsup h_{i}(x)\right] \\
& >-2^{-(n+3)} r \beta_{n+1} \\
& +\sup _{x \in C}\left[\sum_{1}^{n} \beta_{i} g_{i}(x)+\beta_{n+1} h_{n+1}(x)-\left(\sum_{1}^{n+1} \beta_{i}\right) \liminf h_{i}(x)\right] \\
& >\left[\frac{1}{4}+2^{-n}-2^{-(n+2)}\right] r \beta_{n+1}+\sup _{x \in C}\left[\sum_{1}^{n} \beta_{i} g_{i}(x)-\left(\sum_{1}^{n} \beta_{i}\right) \liminf h_{i}(x)\right] \text {. }
\end{aligned}
$$

Now we are prepared to attack property $\mathrm{K}$ when $k=n+1$. Suppose that $\left\{h_{n+1}, \cdots\right\}$ is a sequence of nonoverlapping members of conv $\left\{G_{n+1}^{*}, \cdots\right\}$ and that $\xi$ belongs to $C$ and is such that $h_{n+1}(\xi)-\liminf h_{i}(\xi) \leqq\left[\frac{1}{4}+2^{-(n+1)}\right] r$. Then it is easy to verify directly that

$$
\begin{aligned}
{\left[\frac{1}{4}+2^{-(n+1)}\right] r } & \beta_{n+1} \\
\geqq\left[\sum_{1}^{n} \beta_{i} g_{i}(\xi)\right. & \left.+\beta_{n+1} h_{n+1}(\xi)-\left(\sum_{1}^{n+1} \beta_{i}\right) \limsup h_{i}(\xi)\right] \\
& -\left[\sum_{1}^{n} \beta_{i} g_{i}(\xi)-\left(\sum_{1}^{n} \beta_{i}\right) \liminf h_{i}(\xi)\right] .
\end{aligned}
$$

From (11), it follows that there is a $y$ in $C$ such that

$$
\begin{aligned}
& {\left[\sum_{1}^{n} \beta_{i} g_{i}(y)+\beta_{n+1} h_{n+1}(y)-\left(\sum_{1}^{n+1} \beta_{i}\right) \limsup h_{i}(y)\right]} \\
& >\left[\frac{1}{4}+2^{-n}-2^{-(n+2)}\right] r \beta_{n+1} \\
& +\sup _{x \in C}\left[\sum_{1}^{n} \beta_{i} g_{i}(x)-\left(\sum_{1}^{n} \beta_{i}\right) \lim \inf h_{i}(x)\right] .
\end{aligned}
$$

It follows from (12), (13), and the equality of $\left[\frac{1}{4}+2^{-n}-2^{-(n+2)}\right]$ and $2^{-(n+2)}+\left[\frac{1}{4}+2^{-(n+1)}\right]$, that 


$$
\begin{aligned}
& {\left[\sum_{1}^{n} \beta_{i} g_{i}(y)+\beta_{n+1} h_{n+1}(y)-\left(\sum_{1}^{n+1} \beta_{i}\right) \limsup h_{i}(y)\right]} \\
& \quad>2^{-{ }^{(n+2)} r \beta_{n+1}+\left[\sum_{1}^{n} \beta_{i} g_{i}(\xi)+\beta_{n+1} h_{n+1}(\xi)-\left(\sum_{1}^{n+1} \beta_{i}\right) \lim \sup h_{i}(\xi)\right] .}
\end{aligned}
$$

Therefore property $\mathrm{K}$ is valid when $k=n+1$, for all choices of $\left\{g_{n+1}, \cdots\right\}$ as a sequence of nonoverlapping members of $\operatorname{conv}\left\{G_{n+1}^{*}, G_{n+2}^{*}, \cdots\right\}$.

THEOREM 4. Let $C$ be a bounded, closed, non-weaklv-compact, convex subset of a Banach space $B$. Then there is a continuous linear functional defined on $B$ that does not attain its sup on $C$.

Proof. It follows from Theorem 3 that the number $r$ and the sequences $\left\{z_{i}\right\}$ and $\left\{f_{i}\right\}$ hypothesized for Lemma 2 actually exist. For the resulting sequence $\left\{g_{i}\right\}$, it will be convenient to have

$$
\begin{array}{r}
\left|\sum_{k+1}^{\infty} \beta_{i} g_{i}(y)-\left(\sum_{k+1}^{\infty} \beta_{i}\right) \limsup g_{i}(y)\right|+\left|\sum_{k+1}^{\infty} \beta_{i} g_{i}(\xi)-\left(\sum_{k+1}^{\infty} \beta_{i}\right) g_{i}(\xi)\right| \\
\leqq 2^{-(k+2)} r \beta_{k},
\end{array}
$$

for all $k$ and all $y$ and $\xi$ in $C$. For this, it is sufficient to have

$$
4\left(\sum_{k+1}^{\infty} \beta_{i}\right) M \leqq 2^{-(k+2)} r \beta_{k} \text { or } \sum_{k+1}^{\infty} \beta_{i} \leqq \frac{r \beta_{k}}{2^{k+4} M}
$$

where $M$ is an upper bound for $\{\|x\|: x \in C\}$. This will be satisfied if we let $\beta_{1}=1$ and require that $\beta_{n+1} \leqq 2^{-(n+5)} r \beta_{n} / M$ and $\beta_{n+1} \leqq \frac{1}{2} \beta_{n}$ for all $n$. Now let $\Phi$ be a linear functional of unit norm defined on the space $(m)$ of bounded sequences and such that

$$
\liminf x_{i} \leqq \Phi\left(x_{1}, x_{2}, \cdots\right) \leqq \lim \sup x_{i} .
$$

For example, we can let $\Phi$ be any linear functional of unit norm such that $\Phi\left(x_{1}, x_{2}, \cdots\right)=\lim _{n \rightarrow \infty} x_{n}$ whenever this limit exists (or we could let $\Phi$ be a "Banach limit"-but we do not need the "translation invariance" of a Banach limit). Now for the sequence $\left\{g_{i}\right\}$ of the lemma, define a continuous linear functional $g$ on $B$ by letting $g(z)=\Phi\left[g_{1}(z), g_{2}(z), \cdots\right]$. Then $\|g\| \leqq 1$ and we also have

$$
\liminf g_{i}(z) \leqq g(z) \leqq \limsup g_{i}(z)
$$

for all $z$ in $B$. Let $G$ be defined by

$$
G(x)=\sum_{1}^{\infty} \beta_{i} g_{i}(x)-\left(\sum_{1}^{\infty} \beta_{i}\right) g(x) .
$$

It follows from property $\mathrm{K}$ of the lemma that, if 


$$
g_{k}(\xi)-\liminf g_{i}(\xi) \leqq\left(\frac{1}{4}+2^{-k}\right) r
$$

for some $\xi \in C$ and some $k$, then there is a $y$ in $C$ such that

$$
\begin{aligned}
& {\left[\sum_{1}^{k} \beta_{i} g_{i}(y)-\left(\sum_{1}^{k} \beta_{i}\right) \limsup g_{i}(y)\right]} \\
& >2^{-(k+2)} r \beta_{k}+\left[\sum_{1}^{k} \beta_{i} g_{i}(\xi)-\left(\sum_{1}^{k} \beta_{i}\right) \liminf g_{i}(\xi)\right] .
\end{aligned}
$$

From this and (14) we have

$$
\left[\sum_{1}^{\infty} \beta_{i} g_{i}(y)-\left(\sum_{1}^{\infty} \beta_{i}\right) \limsup g_{i}(y)\right]>\left[\sum_{1}^{\infty} \beta_{i} g_{i}(\xi)-\left(\sum_{1}^{\infty} \beta_{i}\right) \liminf g_{i}(\xi)\right],
$$

and

$$
\sum_{1}^{\infty} \beta_{i} g_{i}(y)-\left(\sum_{1}^{\infty} \beta_{i}\right) g(y)>\sum_{1}^{\infty} \beta_{i} g_{i}(\xi)-\left(\sum_{1}^{\infty} \beta_{i}\right) g(\xi)
$$

or $G(y)>G(\xi)$. Therefore if $G$ attains its $\sup$ on $C$ at $u$, then

$$
g_{k}(u)>\left(\frac{1}{4}+2^{-k}\right) r+\liminf g_{i}(u) \quad \text { for all } k .
$$

This implies that $\liminf g_{i}(u) \geqq \frac{1}{4} r+\liminf g_{i}(u)$, a clear contradiction.

The first of the following generalizations of Theorem 4 was suggested by Isaac Namioka, the second by N. T. Peck.

THEOREM 5. A weakly closed subset $S$ of a Banach space B is weakly compact if and only if each continuous linear functional on B attains its sup on $S$.

Proof. For any subset $S$ of $B$ and any continuous linear functional $f$, the sup of $f$ on $S$ is equal to the sup of $f$ on the closed convex span of $S$. Therefore if $S$ is weakly closed and each continuous linear functional on $B$ attains its sup on $S$, then the closed convex span of $S$ is weakly compact [Theorem 4] and $S$ is weakly compact. It follows directly from the definition of weak sequential compactness that if a subset $S$ is weakly compact, then each continuous linear functional attains its sup on $S$.

THEOREM 6. If a subset $S$ of a complete locally convex space $E$ is such tha every continuous linear functional on $E$ attains its sup on $S$, then the weak closure of $S$ is weakly compact.

Proof. The weak closure of a set $S$ will be denoted by wcl(S). Note that the sup of a continuous linear functional on $\operatorname{wcl}(S)$ is equal to its sup on $S$. As is well known [4, pp. 46-47], there is a family $\left\{B_{a}: a \in A\right\}$ of Banach spaces and a 
linear homeomorphism $h$ that carries $E$ onto a closed linear subspace $E^{\prime}$ of the product space $P=\prod_{a \in A} B_{a}$. If $w$ indicates the weak topology, then $\left(E^{\prime}, w\right)$ is a closed subspace of $(P, w)$ and $(P, w)=\prod_{a \in A}\left(B_{a}, w\right)$.

For each $a \subset A$, let $\pi_{a}$ denote the canonical projection of $P$ onto $B_{a}$. If $f_{a}$ is continuous linear functional on $B_{a}$, then $f_{a} \pi_{a} h$ is a continuous linear functional on $E$. Since $f_{a} \pi_{a} h$ attains its sup on $S, f_{a}$ attains its sup on $\pi_{a} h S$. From Theorem 5 it follows that wcl $\left(\pi_{a} h S\right)$ is weakly compact, whence of course the set $\prod_{a \leq A} \mathrm{wcl}\left(\pi_{a} h S\right)$ is a weakly compact subset of $P$ and its weakly closed subset wcl $(h S)$ is also weakly compact. But then wcl(S) is weakly compact and the proof is complete.

\title{
REFERENCES
}

1. W. F. Eberlein, Weak compactness in Banach spaces. I, Proc. Nat. Acad. Sci. U.S.A. 33 (1947), 51-53.

2. R. C. James, Reflexivity and supremum of linear functionals, Ann. of Math. (2) 66(1957), 159-169.

3. - Characterizations of reflexivity, Studia Math. 23 (1964), 205-216.

4. J. L. Kelley and I. Namioka, Linear topological spaces, Van Nostrand, Princeton, N. J., 1963.

5. V. L. Klee, Jr., A conjecture on weak compactness, Trans. Amer. Math. Soc. 104(1962), 398-402.

6. V. Smulian, On the principle of inclusion in the space of type (B), Rec. Math. (Mat. Sb. (N. S.)) 5 (1939), 317-328.

7. J.W. Tukey, Some notes on the separation of convex sets, Portugal. Math. 3 (1942), 95-102.

\author{
HaRvey Mudd College, \\ Claremont, California \\ INSTITUTE FOR ADVANCED STUDY, \\ Princeton, New Jersey
}

